\title{
Association of Maternal and Umbilical Cord Blood Lipid Parameters with Uterine and Fetal-Placental Blood Flow in Hypertensive and Normotensive Pregnancies
}

This article was published in the following Dove Press journal:

International Journal of Women's Health

\section{Kamran Hessami ${ }^{1,2}$ \\ Maryam Kasraeian ${ }^{2}$ \\ Nasrin Asadi ${ }^{2}$ \\ Homeira Vafaei $\mathbb{I D}^{2}$ \\ Leila Foroughinia $^{3}$}

'Student Research Committee, Shiraz University of Medical Sciences, Shiraz, Iran; ${ }^{2}$ Maternal-Fetal Medicine Research Center, Shiraz University of Medical Sciences, Shiraz, Iran; ${ }^{3}$ Eastern Health, Box Hill Hospital, FRANZCOG, Box Hill, Victoria, Australia
Correspondence: Kamran Hessami Maternal-Fetal Medicine (Perinatology) Research Center, Hafez Hospital, Chamran Ave, Shiraz, Iran

Tel +98-7I-36I28257

Fax +98-7I-362I28257

Email hessamikamran@gmail.com
Purpose: There is a growing evidence suggesting that hypertensive disorders of pregnancy (HDP), especially preeclampsia, are associated with an increased risk of cardiometabolic disease for both mother and child later in life. The objective of this study was to determine the association of maternal and umbilical cord blood (UCB) lipid profiles with uterine and fetal-placental blood flow at the third trimester of pregnancy.

Patients and Methods: A total of 1,135 women were prospectively followed through pregnancy and data for 812 mother-newborn pairs, including 170 HDP developed subjects and 642 normotensive subjects were analyzed at the end of the study. Maternal serum and UCB triglyceride (TG), total cholesterol (TC), high-density lipoprotein cholesterol (HDL-C), low-density lipoprotein cholesterol (LDL-C), and non-HDL-C levels were compared between HDP and normotensive groups; moreover, the association between lipid parameters and abnormal pulsatility indices (PIs) of uterine (UtA), umbilical (UA), and middle cerebral (MCA) arteries was evaluated with multivariate regression analysis models. The same analyses were carried out on subgroups of HDP (preeclampsia vs gestational hypertension). Results: The mean TG, TC, LDL-C, and non-HDL-C levels were significantly higher in mother-newborn pairs of the HDP group compared to the normotensive group. In the HDP group, maternal TG and non-HDL-C levels were shown to have a significant association with abnormal UtA-PI ( $\mathrm{p}<0.001$ and $\mathrm{p}=0.039$, respectively). We also found a positive significant association of fetal hypertriglyceridemia with abnormal UA-PI and MCA-PI in the HDP group ( $\mathrm{p}=0.042$ and $\mathrm{p}=0.021$, respectively). However, no such associations were observed in normotensive mother-newborn pairs. Similar trends were observed in preeclamptic subjects after subgroup analysis.

Conclusion: Maternal TG and non-HDL-C levels as well as fetal TG level are significantly associated with disturbed uterine and fetal-placental blood flow in HDP.

Keywords: preeclampsia, gestational hypertension, uterine artery, umbilical artery, middle cerebral artery, lipid

\section{Introduction}

Pregnancy is a unique period in life associated with metabolic changes in order to ensure an adequate supply of energy source including glucose, amino acids, and lipids, to the developing fetus. Maternal blood lipid concentrations increase progressively with advancing gestation, which is considered a physiological adaptation 
to normal pregnancy. ${ }^{1,2}$ However, exaggerated elevations in maternal blood cholesterol and/or triglyceride levels during pregnancy apparently are associated with preterm birth, ${ }^{3}$ gestational diabetes mellitus (GDM), ${ }^{3,4}$ hypertensive disorders of pregnancy (HDP), ${ }^{4-7}$ macrosomia, ${ }^{4}$ and also an increased risk of atherosclerotic events later in life for both mothers and their babies. ${ }^{3,8}$

In a meta-analysis, ${ }^{9}$ it was demonstrated that maternal triglyceride (TG), total cholesterol (TC), high-density lipoprotein cholesterol (HDL-C), and non-HDL-C levels during pregnancy are significantly associated with the risk of preeclampsia, which is the main subtype of HDP. While, the association between low-density lipoprotein cholesterol (LDL-C) and preeclampsia was not conclusive. ${ }^{9}$ A growing number of studies suggest that dyslipidemia during pregnancy especially in those complicated with preeclampsia may play a role in endothelial dysfunction and the subsequent atherosclerosis formation. ${ }^{10,11}$ Therefore, we hypothesized that the latter process may be present upon diagnosing preeclampsia and there may be a pathophysiological link between abnormal lipid levels and disturbed uteroplacental blood flow during pregnancy. Accordingly, it was suggested that increased maternal blood TG and TC/HDL-C ratio could potentially worsen the uterine blood flow assessed by Doppler ultrasound in pregnancies complicated with $\mathrm{GDM} ;{ }^{12}$ however, there is no large-scale analysis of this association for patients with HDP.

Third-trimester Doppler flow velocity waveform analysis is a noninvasive and useful method for assessing fetal growth. Additionally, it has been used successfully for prediction of perinatal morbidity and mortality among highrisk pregnancies, such as those complicated with HDP. ${ }^{13,14}$ This method is clinically important because the impaired uterine and/or fetal placental blood perfusion caused by vascular dysfunction precedes clinical manifestations of HDP $;{ }^{15}$ therefore, this would provide enough time for clinicians to apply the most appropriate treatment options.

To date, there is no evidence of the association of maternal and umbilical cord blood (UCB) lipid parameters with uterine and fetal-placental blood flow, neither in hypertensive nor in normotensive pregnancies. In addition, most studies compare the lipid levels between normotensive and hypertensive women without considering the preconception lipid status of study subjects, which may lead to a false conclusion; for example, a proportion of patients may already have dyslipidemia and associated vascular dysfunction before conception, which is not attributable to the HDP.
The objectives of this study were as follows: 1) assessing the link between HDP-induced blood flow changes in the mother, revealed as changes in the uterine artery pulsatility index (UtA-PI), with maternal blood lipid levels; 2) assessing the link between HDP-induced blood flow changes in the fetus, revealed as changes in the umbilical and middle cerebral arteries pulsatility indices (UA-PI and MCA-PI), with UCB lipid levels; 3) comparing and showing whether these relationships are significantly different between hypertensive and normotensive pregnancies; and 4) conducting a separate analysis regarding this association on subgroups of the HDP group (preeclampsia vs gestational hypertension).

\section{Methods and Materials Study Population}

Pregnant women who had their prenatal care and delivery at Hafez and Hazrat Zeinab Hospitals affiliated to Shiraz University of Medical Sciences were prospectively recruited for the present study from January 2018 to August 2019. All participants provided the study with written informed consent. The study complies with the Declaration of Helsinki and was approved by the Institutional Review Board of Shiraz University of Medical Sciences, Shiraz, Iran (97-0101-18902).

The inclusion criteria were as follows: 1) maternal age of 20-35 years; 2) singleton pregnancies; 3 ) naturally fertilized; and 4) having complete medical records and a clear gestational age confirmed by the first trimester ultrasound. Exclusion criteria of the pregnant women were as follows: 1) women with chronic medical conditions that may affect the lipid profile such as pre-existing diabetes mellitus, chronic hypertension, thyroid diseases, renal diseases, autoimmune disorders etc.; 2) polycystic ovary syndrome; 3 ) preconception $\mathrm{BMI} \geq 30$; 4) fetal/neonatal death; 5) nonfasting state at the time of maternal blood sampling (less than 8 hours); 6) intrauterine fetal growth restriction (IUGR), which may occur simultaneously with HDP and negatively affects UA-PI and MCA-PI; 7) pregnancies with GDM were also excluded due to the possibility of increasing maternal lipid levels and disturbing uterine blood perfusion during pregnancy; ${ }^{12}$ and 8 ) delivery $\leq 30$ weeks of gestation. Also, mothers aged $>35$ years were not included in the study because of their potential to have disturbed uterine blood flow possibly leading to bias. ${ }^{16}$

During the study period, 1,240 women were initially enrolled. We excluded 428 participants for the following reasons: pre-existing dyslipidemia at the first antenatal 
visit $(n=63)$, pre-existing chronic diseases $(n=42)$, fetal/ neonatal death $(n=15)$, IUGR and/or GDM $(n=182)$, failing in follow-up or delivery at another healthcare facility $(n=87)$, missing data on maternal or UCB lipid levels $(n=39)$. Finally, 812 pairs of mothers and newborns remained for analysis in the current report (Figure 1).

\section{Data Collection and Measurements}

All data about maternal age, body mass index (BMI), obstetrical history, gestational age (confirmed by the first trimester ultrasound), and lipid status of patients was collected at the first antenatal visit (ranging from 8 to 14 weeks of gestation). Women with previously known dyslipidemia were not included in the study. However, serum lipid profile was screened for women with unknown status of lipid profile before entry to the study cohort and individuals with abnormal lipid parameters according to new recommendations from the American Heart Association were considered ineligible for recruitment. ${ }^{17}$
The study population was followed from recruitment at the first antenatal visit to 28 days postpartum to ensure neonatal survival. Blood pressure measurement, urine dipstick test, weight gain measurement, and other routine examinations were performed at each antenatal visit. Women with blood pressure of $\geq 140 / 90 \mathrm{~mm} \mathrm{Hg}$, measured twice at least 6 hours apart, after 20 weeks of gestation were considered as the gestational hypertension group. Additionally, patients who met the abovementioned criteria plus proteinuria $\geq 300 \mathrm{mg}$ in 24 hour urine or $\geq 1+$ using a urine dipstick were considered as the preeclampsia group. At the end of the study, participants were grouped into one of the following groups: HDP $(n=170)$, including preeclampsia $(n=80)$ and gestational hypertension $(\mathrm{n}=90)$, and normotensive uncomplicated pregnancies $(n=642)$.

Maternal blood samples were taken after overnight fasting from all mothers at the third trimester of pregnancy ( $\geq 28$ weeks of gestation). The study population was

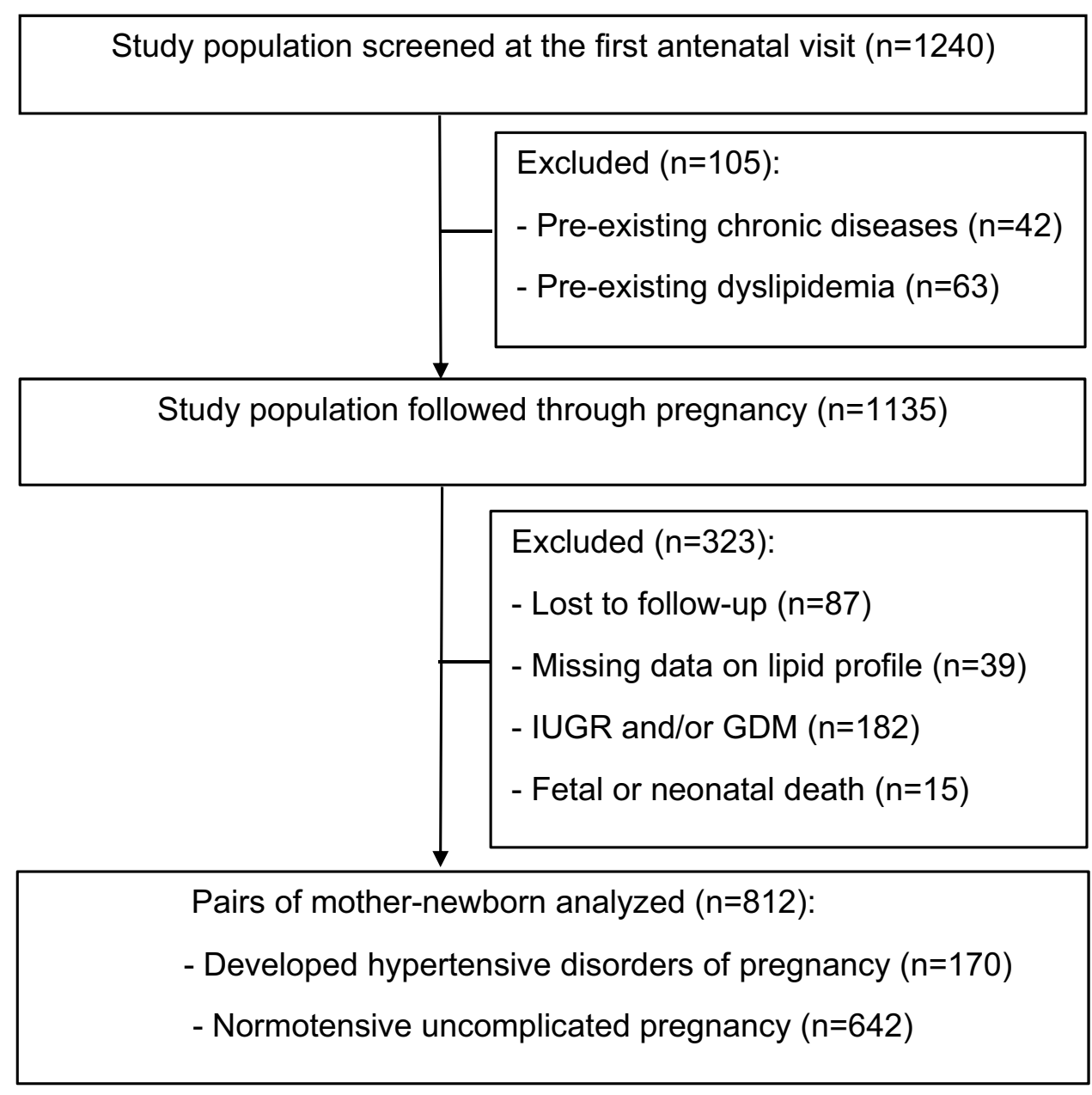

Figure I Schematic presentation of the study cohort. 
followed to the delivery and blood samples were taken from the umbilical cord immediately after delivery. The serum samples were separated by centrifugation at $3500 \mathrm{rpm}$ for 10 minutes and then serum samples were stored at $-70^{\circ} \mathrm{C}$ immediately after centrifugation until further analysis. Serum TC, TG, HDL-C, and LDL-C was analyzed by the enzymatic colorimetric method (Pars Azmoon, Tehran, Iran) and non-HDL-C was calculated as TC (mg/dL) - HDL-C (mg/dL).

\section{Doppler Ultrasound Examination}

Doppler velocimetry was performed using a Voluson E8 Sonography machine (General Electronic Healthcare Technologies, Wisconsin, USA). All sonographic data collection was done within 28-34 weeks of gestation by an expert sonographer to eliminate inter-observer variability. In order to assess UtA-PI, the probe was placed on the lower quadrants of the abdomen and angled medially, then color Doppler imaging was employed to identify the UtA at the point where it crossed the external iliac artery. Right and left UtA-PI were measured twice with a 5-minute interval, and the mean of the four UtA-PI values (two values for each artery) was defined as mean UtA-PI. Doppler flow spectra of UA and MCA were obtained from the free loop portion of the umbilical cord and the distal portion of the MCA by methods that have been previously described. ${ }^{18} \mathrm{PI}$ values above the 95 th percentile standardized for the gestational age were set as abnormal for the UtA and UA, ${ }^{19,20}$ and below the 10th percentile for the $\mathrm{MCA}^{20}$

\section{Statistical Analysis}

Statistical analyses were performed with SPSS version 21.0 (SPSS Inc., IBM Corporation, Armonk, NY, USA). The data were expressed as the means (SD) or frequency (percentage), respectively. Differences between baseline characteristics of study groups were evaluated by Student's $t$-test or chi-square test accordingly. Multivariate logistic regression analyses were performed to test the association between: 1) maternal blood lipid parameters and abnormal UtA-PI after adjustment for potentially confounding risk factors (Model1: maternal age, systolic and diastolic blood pressure, preconception BMI, weight gain during pregnancy, parity, and gestational age at the time of blood sampling); 2) UCB lipid parameters and abnormal UA-PI and MCA-PI after adjustment for potentially confounding risk factors (Model-2: all of the risk factors adjusted in Model-1 in addition to gender of fetus, gestational age at birth, anthropometric measurements of newborn at birth, and maternal blood lipid parameters). The lipid parameters that were significantly associated with the presence of abnormal PI were categorized into quartiles, and the association between the presence of abnormal PI and the different lipid quartiles, with the lowest quartile as reference, was analyzed. All statistical tests were two tailed, and p-values $<0.05$ were considered significant.

\section{Results}

\section{Baseline Characteristics, Lipid Parameters, and Doppler Indices}

Table 1 presents maternal and neonatal characteristics of our study cohort. Among the 812 eligible women in the present study, the mean (SD) maternal age and preconception BMI were 28.07 (4.97) years old and $23.05(1.64) \mathrm{kg} / \mathrm{m}^{2}$, respectively. The rate of nulliparity was $49.8 \%$ in the study population. Nulliparity was more common among the HDP group than among the normotensive women $(60 \%$ vs $47.04 \%$, $\mathrm{p}=0.003$ ). The mean (SD) gestational age of the HDP group at delivery was 37.14 (1.97) weeks, while in the normotensive group it was $38.66(1.60)$ weeks $(p<0.001)$. Consequently, first- and fifth-minute APGAR scores, birth weight, height, abdominal circumference, and head circumference in newborns of the HDP group were significantly lower than those in the normotensive group.

Table 2 summarizes the Doppler findings, maternal, UCB lipid profiles, and their comparison between study groups. The mean UtA-PI and UA-PI was significantly higher in the HDP than normotensive group $(\mathrm{p}<0.001)$, whereas the mean MCA-PI was lower in pregnancies with HDP than normotensive pregnancies $(\mathrm{p}<0.001)$. We found that maternal and UCB levels of TG, TC, LDL-C, and non-HDL-C were significantly higher in the HDP group compared to the normotensive group. UCB level of HDL-C was significantly lower in the HDP group than in the normotensive group, while maternal HDL-C level was comparable between the study groups.

\section{Association of Abnormal Pulsatility Indices (PIs) of UtA, UA and MCA with Lipid Markers}

After adjustment for potential confounding factors of Model 1 (as discussed above), maternal TG exhibits the strongest association with abnormal UtA-PI ( $\mathrm{p}<0.001, \mathrm{AOR}=1.033,95 \%$ CI: $1.020-1.045)$, followed by non-HDL level $(\mathrm{p}=0.039$, $\mathrm{AOR}=1.011,95 \% \mathrm{CI}: 1.001-1.021)$ in women with HDP, 
Table I Maternal and Neonatal Characteristics of Study Population

\begin{tabular}{|c|c|c|c|c|}
\hline & All Subjects $(N=8 \mid 2)$ & $\operatorname{HDP}(n=\mid 70)$ & Normotensive $(n=642)$ & p-value* \\
\hline \multicolumn{5}{|l|}{ Maternal Variables } \\
\hline Maternal age, years & $28.07(4.97)$ & $27.86(5.02)$ & $28.13(4.96)$ & 0.530 \\
\hline Preconception BMI, kg/m² & $23.05(1.64)$ & $23.04(1.80)$ & $23.05(1.59)$ & 0.936 \\
\hline BMI before delivery, $\mathrm{kg} / \mathrm{m}^{2}$ & $27.85(1.72)$ & $27.93(1.96)$ & $27.82(1.65)$ & 0.488 \\
\hline Weight gain during pregnancy, kg & $12.27(1.29)$ & $12.52(1.34)$ & $\mid 2.21(1.28)$ & 0.006 \\
\hline $\mathrm{SBP}, \mathrm{mmHg}$ & $129.06(15.86)$ & I49.83 (16.97) & I $23.55(9.88)$ & $<0.001$ \\
\hline $\mathrm{DBP}, \mathrm{mmHg}$ & $77.83(9.32)$ & $87.14(11.31)$ & $75.36(6.86)$ & $<0.001$ \\
\hline \multicolumn{5}{|l|}{ Parity: } \\
\hline Nulliparous & $404(49.8 \%)$ & $102(60 \%)$ & 302 (47.04\%) & 0.003 \\
\hline Multiparous & $408(50.2 \%)$ & $68(40 \%)$ & $340(52.96 \%)$ & \\
\hline Cesarean section delivery & $278(34.2 \%)$ & 99 (58.2\%) & 179 (27.9\%) & $<0.001$ \\
\hline \multicolumn{5}{|l|}{ Neonatal Variables } \\
\hline Gestational age, weeks & $38.34(1.79)$ & $37.14(1.97)$ & $38.66(1.60)$ & $<0.001$ \\
\hline Gender (boy) & 427 (52.59\%) & 94 (55.29\%) & $333(51.87 \%)$ & 0.426 \\
\hline First-minute APGAR & $8.32(1.23)$ & $7.54(1.43)$ & $8.53(1.09)$ & $<0.001$ \\
\hline Fifth-minute APGAR & $9.31(1.05)$ & $8.76(1.33)$ & $9.45(0.91)$ & $<0.001$ \\
\hline Weight, gr & $2892.94(288.30)$ & $2831.92(305.37)$ & $2909.10(281.64)$ & 0.003 \\
\hline Height, cm & $48.60(2.80)$ & $46.84(3.22)$ & $49.07(2.48)$ & $<0.001$ \\
\hline Abdominal circumference, $\mathrm{cm}$ & $31.62(3.00)$ & $29.98(2.96)$ & $32.05(2.86)$ & $<0.001$ \\
\hline Head circumference, $\mathrm{cm}$ & $34.20(1.35)$ & $33.64(1.50)$ & $34.34(1.27)$ & $<0.001$ \\
\hline
\end{tabular}

Notes: *P-values were derived from the comparisons between the HDP and normotensive groups.

Abbreviations: HDP, hypertensive disorders of pregnancy; BMI, body mass index; SBP, systolic blood pressure; DBP, diastolic blood pressure.

Table 2 Doppler Indices and Lipid Parameters of the Study Population

\begin{tabular}{|c|c|c|c|c|}
\hline & All Subjects $(\mathbf{N}=\mathbf{8} \mid \mathbf{2})$ & HDP $(n=\mid 70)$ & Normotensive $(n=642)$ & \multirow[t]{2}{*}{ p-value } \\
\hline & Mean (SD) & Mean (SD) & Mean (SD) & \\
\hline \multicolumn{5}{|l|}{ Doppler Indices } \\
\hline UtA-PI & $0.77(0.17)$ & $0.91(0.17)$ & $0.73(0.15)$ & $<0.001$ \\
\hline UA-PI & $0.77(0.16)$ & $0.92(0.17)$ & $0.73(0.14)$ & $<0.001$ \\
\hline MCA-PI & $\mathrm{I} .73(0.25)$ & $1.61(0.25)$ & $1.77(0.23)$ & $<0.001$ \\
\hline \multicolumn{5}{|c|}{ Maternal Lipid Parameters } \\
\hline $\mathrm{TG}, \mathrm{mg} / \mathrm{dL}$ & $215.12(40.86)$ & $236.15(46.81)$ & $209.55(37.23)$ & $<0.001$ \\
\hline $\mathrm{TC}, \mathrm{mg} / \mathrm{dL}$ & $224.80(33.81)$ & $240.14(43.44)$ & 220.74 (29.49) & $<0.001$ \\
\hline $\mathrm{HDL}-\mathrm{C}, \mathrm{mg} / \mathrm{dL}$ & $50.15(7.22)$ & $50.32(8.69)$ & $50.11(6.78)$ & 0.734 \\
\hline LDL-C, mg/dL & $131.69(29.94)$ & $|42.4|(36.73)$ & $128.85(27.20)$ & $<0.001$ \\
\hline Non-HDL-C, mg/dL & $174.97(33.66)$ & $190.17(41.37)$ & $170.95(30.07)$ & $<0.001$ \\
\hline \multicolumn{5}{|c|}{ UCB Lipid Parameters } \\
\hline $\mathrm{TG}, \mathrm{mg} / \mathrm{dL}$ & $44.06(14.04)$ & $50.81(19.93)$ & $42.25(11.35)$ & $<0.001$ \\
\hline $\mathrm{TC}, \mathrm{mg} / \mathrm{dL}$ & $61.77(11.39)$ & $63.64(12.90)$ & $61.27(10.90)$ & 0.016 \\
\hline HDL-C, mg/dL & $27.68(5.73)$ & $26.46(6.60)$ & $28.01(5.43)$ & 0.002 \\
\hline LDL-C, mg/dL & $25.34(9.27)$ & $27.32(9.54)$ & $24.81(9.13)$ & 0.002 \\
\hline Non-HDL-C, mg/dL & $34.09(9.68)$ & $37.18(10.13)$ & $33.26(9.40)$ & $<0.001$ \\
\hline
\end{tabular}

Notes: *P-values were derived from the comparisons between the HDP and normotensive groups.

Abbreviations: HDP, hypertensive disorders of pregnancy; UtA-PI, uterine artery pulsatility index; UA-PI, umbilical artery pulsatility index; MCA-PI, middle cerebral artery pulsatility index; TG, triglyceride; TC, total cholesterol; HDL-C, high density lipoprotein cholesterol; LDL-C, low density lipoprotein cholesterol; Non-HDL-C, non high density lipoprotein cholesterol; UCB, umbilical cord blood. 
Table 3 Adjusted Odd Ratios for Abnormal Doppler According to Lipid Parameters in the Study Population

\begin{tabular}{|c|c|c|c|c|}
\hline & \multicolumn{2}{|l|}{ HDP $(n=170)$} & \multicolumn{2}{|c|}{ Normotensive $(n=642)$} \\
\hline & OR $(95 \% \mathrm{Cl})$ & p-value & OR $(95 \% \mathrm{Cl})$ & p-value \\
\hline \multicolumn{5}{|c|}{ Association between maternal lipid parameters and abnormal UtA-PI } \\
\hline TG & $1.033(1.020-1.045)$ & $<0.00 I^{*}$ & $1.007(0.999-1.015)$ & 0.072 \\
\hline TC & $1.009(0.999-1.019)$ & 0.065 & $1.004(0.993-1.014)$ & 0.497 \\
\hline HDL-C & $0.999(0.957-1.044)$ & 0.977 & $0.977(0.934-1.022)$ & 0.308 \\
\hline LDL-C & $1.003(0.993-1.014)$ & 0.532 & $1.003(0.991-1.014)$ & 0.656 \\
\hline Non-HDL-C & $1.011(1.001-1.021)$ & $0.039 *$ & 1.009 (0.999-1.018) & 0.066 \\
\hline \multicolumn{5}{|c|}{ Association between UCB lipid parameters and abnormal UA-PI ${ }^{b}$} \\
\hline TG & $1.028(1.001-1.053)$ & $0.042^{*}$ & $1.025(0.934-1.125)$ & 0.597 \\
\hline TC & 1.031 (0.984-1.080) & 0.196 & I.024 (0.890-I.I77) & 0.744 \\
\hline HDL-C & $0.987(0.9 \mid 4-1.065)$ & 0.735 & I.I75 (0.888-I.555) & 0.258 \\
\hline LDL-C & $1.024(0.967-1.083)$ & 0.420 & $0.968(0.839-1.117)$ & 0.659 \\
\hline Non-HDL-C & $1.053(0.996-|| I \mid 2)$. & 0.067 & $0.975(0.846-1.124)$ & 0.728 \\
\hline \multicolumn{5}{|c|}{ Association between UCB lipid parameters and abnormal MCA-PI } \\
\hline TG & $1.021(1.003-1.039)$ & $0.021 *$ & $1.015(0.989-1.041)$ & 0.275 \\
\hline $\mathrm{TC}$ & $0.991(0.945-1.039)$ & 0.702 & $0.98 I(0.940-1.024)$ & 0.377 \\
\hline HDL-C & $0.960(0.878-1.050)$ & 0.377 & $1.038(0.946-1.139)$ & 0.433 \\
\hline LDL-C & 0.991 (0.930-1.057) & 0.788 & 0.951 (0.897-I.008) & 0.090 \\
\hline Non-HDL-C & $1.004(0.944-1.067)$ & 0.903 & $0.963(0.917-1.011)$ & 0.127 \\
\hline
\end{tabular}

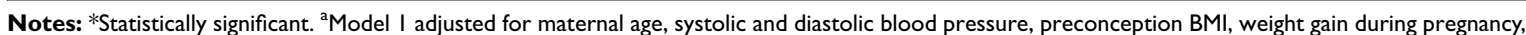
parity, and gestational age at the time of blood sampling. 'Model 2 adjusted for all the risk factors adjusted in Model I in addition to gender of fetus, anthropometric measurements of newborn at birth, and maternal blood lipid parameters.

Abbreviations: HDP, hypertensive disorders of pregnancy; UtA-PI, uterine artery pulsatility index; UA-PI, umbilical artery pulsatility index; MCA-PI, middle cerebral artery pulsatility index; TG, triglyceride; TC, total cholesterol; HDL-C, high density lipoprotein cholesterol; LDL-C, low density lipoprotein cholesterol; Non-HDL-C, non high density lipoprotein cholesterol; UCB, umbilical cord blood.

while no such association was observed in normotensive subjects (Table 3). Moreover, after adjustment for potential confounding factors of Model 2 (as discussed above), UCB TG showed a significant association with abnormal UA-PI $(p=0.042, A O R=1.028,95 \%$ CI: $1.001-1.053)$ and MCAPI $(p=0.021, A O R=1.021,95 \%$ CI: $1.003-1.039)$ in the HDP group (Table 3 ).

\section{Association of Abnormal Pulsatility Indices of UtA, UA and MCA with Lipid Quartiles}

The lipid parameters that showed significant association with abnormal PI in the HDP group (maternal TG, nonHDL-C, and UCB TG) were categorized into quartiles and the association between each quartile and the presence of abnormal PI was evaluated, with the lowest quartile as reference (Figure 2). Compared to the lowest TG and nonHDL-C quartiles, subjects with the highest TG and nonHDL-C quartiles had significantly higher adjusted odds ratios $(\mathrm{AOR})(\mathrm{AOR}=2.688$ and $\mathrm{AOR}=3.411$, respectively $)$ for abnormal UtA-PI after adjusting for confounding factors in Model 1 (Figure 2A and B). The third and fourth quartiles of UCB TG were associated with a higher risk of abnormal UA-PI compared to the lowest quartile $(\mathrm{AOR}=3.071$ and $\mathrm{AOR}=3.256$, respectively). In the meanwhile, study subjects with UCB TG in the fourth quartile had significantly increased risk of abnormal MCA-PI (AOR $=4.115$, $\mathrm{p}=0.014$ ) (Figure 2C and D). Consequently, there was a dose-response association between maternal TG, nonHDL-C and UCB TG and odds ratio of abnormal uterine or fetal-placental blood flow.

\section{Separate Analysis on Subgroups of HDP (Preeclampsia and Gestational Hypertension)}

We conducted a separate analysis on the study subjects with HDP to find out whether there is a similar association between lipid levels and abnormal blood flow in preeclampsia $(n=80)$ and/or gestational hypertension $(n=90)$ subgroups. In preeclamptic patients, maternal TG, non-HDL 

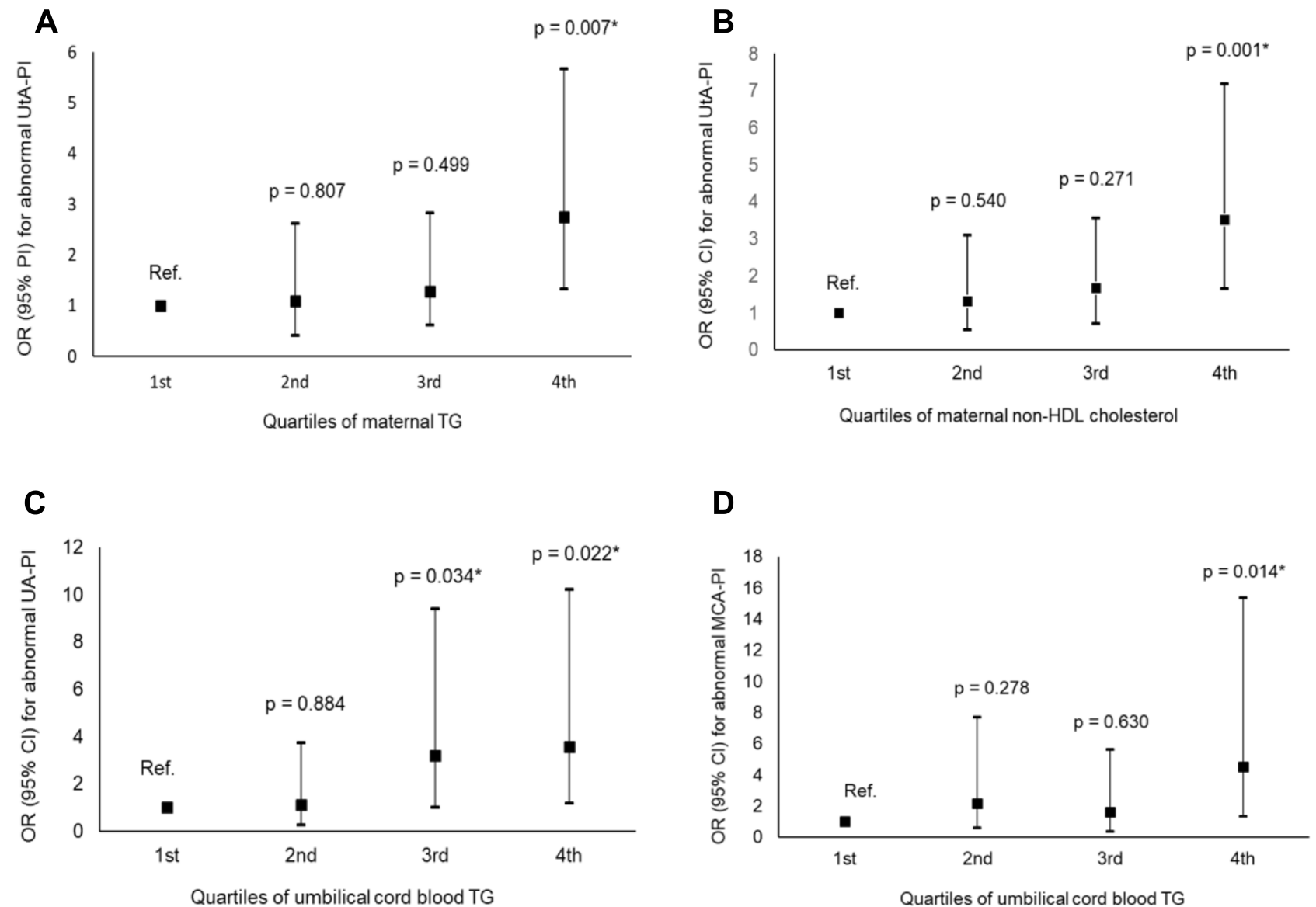

Figure 2 (A) Adjusted odds ratios (OR) for abnormal UtA-PI across quartiles of maternal TG. (B) Adjusted odds ratios (OR) for abnormal UtA-PI across quartiles of maternal non-HDL-c. (C) Adjusted odds ratios (OR) for abnormal UA-PI across quartiles of UCB-TG. (D) Adjusted odds ratios (OR) for abnormal MCA-Pl across quartiles of UCB-TG. *Statistically significant.

Abbreviations: UtA-PI, uterine artery pulsatility index; UA-PI, umbilical artery pulsatility index; MCA-PI, middle cerebral artery pulsatility index; TG, triglycerides; nonHDL, non-high-density lipoprotein.

-C, and UCB TG levels remained significantly associated with abnormal UtA-PI and UA-PI, respectively (Table 4). In addition to TG and non-HDL-C, maternal TC was shown to be significantly associated with abnormal UtA-PI in preeclampsia $(\mathrm{AOR}=1.015, \mathrm{p}=0.034)$, while in patients with gestational hypertension only maternal TG level was significantly associated with abnormal UtA-PI and no association was observed between lipid parameters and abnormal UA-PI or MCA-PI (Table 4).

\section{Discussion}

This is the first study to assess lipid risk factors for disturbed uteroplacental blood flow in a pregnant cohort that included both hypertensive and normotensive participants. We noted that maternal serum TG and non-HDL-C concentrations in late pregnancy were independently and positively associated with the risk of disturbed UtA blood flow. Accordingly, there was a dose-response relationship between quartiles of maternal TG and non-HDL-C and the presence of an abnormal UtA-PI, further reinforcing the strength of these findings. Additionally, an increased UAPI was correlated with fetal hypertriglyceridemia in the HDP group. The association patterns for these lipid parameters and Doppler indices showed a similar trend when preeclamptic subjects (vs gestational hypertension) were analyzed separately, suggesting that preeclampsiarelated metabolic changes may play a significant role in deteriorating uteroplacental blood flow.

Although there is a great inconsistency and even contradictory results in the literature about lipid markers in preeclampsia, a recent meta-analysis showed that elevated TC, non-HDL-C, and TG levels are associated with preeclampsia, ${ }^{9}$ which is partly in line with the findings of the present study. Now, it is well elucidated that preeclampsia is associated with metabolic aberrations; however, scholars suggest that further investigations are 
Table 4 Adjusted Odd Ratios for Abnormal Doppler According to Lipid Parameters in Hypertensive Subgroups

\begin{tabular}{|c|c|c|c|c|}
\hline & \multicolumn{2}{|c|}{ Preeclampsia $(n=80)$} & \multicolumn{2}{|c|}{ Gestational Hypertension $(n=90)$} \\
\hline & OR $(95 \% \mathrm{Cl})$ & p-value & OR $(95 \% \mathrm{Cl})$ & p-value \\
\hline \multicolumn{5}{|c|}{ Association between maternal lipid parameters and abnormal UtA-PI } \\
\hline TG & $1.032(1.015-1.049)$ & $<0.00 I^{*}$ & $1.035(1.013-1.058)$ & $0.002 *$ \\
\hline TC & $1.015(1.001-1.029)$ & $0.034 *$ & $0.997(0.981-1.013)$ & 0.674 \\
\hline HDL-C & $1.027(0.969-1.089)$ & 0.372 & $0.929(0.852-1.012)$ & 0.092 \\
\hline LDL-C & $1.010(0.994-1.025)$ & 0.214 & $0.988(0.969-1.008)$ & 0.248 \\
\hline Non-HDL-C & $1.016(1.001-1.032)$ & $0.031 *$ & $0.999(0.983-1.016)$ & 0.936 \\
\hline \multicolumn{5}{|c|}{ Association between UCB lipid parameters and abnormal UA-PI } \\
\hline TG & $1.084(1.010-1.164)$ & $0.026 *$ & $1.019(0.980-1.059)$ & 0.346 \\
\hline $\mathrm{TC}$ & $1.022(0.952-1.098)$ & 0.544 & $1.230(0.988-|.53|)$ & 0.064 \\
\hline HDL-C & $0.984(0.883-1.097)$ & 0.775 & I.I05 (0.887-I.377) & 0.374 \\
\hline LDL-C & $1.009(0.923-1.104)$ & 0.842 & 1.081 (0.936-I.249) & 0.290 \\
\hline Non-HDL-C & $1.040(0.956-|| 3 \mid)$. & 0.359 & I.I78 (0.99I-I.399) & 0.063 \\
\hline \multicolumn{5}{|c|}{ Association between UCB lipid parameters and abnormal MCA-PI } \\
\hline TG & $1.062(0.956-1.180)$ & 0.266 & $0.998(0.916-1.087)$ & 0.964 \\
\hline $\mathrm{TC}$ & $1.008(0.906-1.122)$ & 0.879 & $0.932(0.850-1.022)$ & 0.137 \\
\hline HDL-C & $0.976(0.849-1.122)$ & 0.735 & $0.900(0.743-1.090)$ & $0.28 I$ \\
\hline LDL-C & $1.014(0.893-1.15 I)$ & 0.832 & $0.914(0.807-1.034)$ & 0.153 \\
\hline Non-HDL-C & $1.029(0.91 I-1.16 I)$ & 0.650 & $0.918(0.814-1.035)$ & 0.162 \\
\hline
\end{tabular}

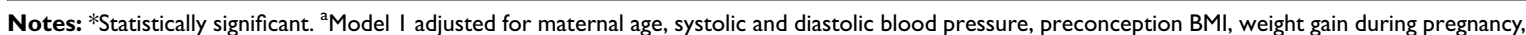
parity, and gestational age at the time of blood sampling. 'Model 2 adjusted for all of the risk factors adjusted in Model I in addition to gender of fetus, anthropometric measurements of newborn at birth, and maternal blood lipid parameters.

Abbreviations: UtA-PI, uterine artery pulsatility index; UA-PI, umbilical artery pulsatility index; MCA-PI, middle cerebral artery pulsatility index; TG, triglyceride; TC, total cholesterol; HDL-C, high density lipoprotein cholesterol; LDL-C, low density lipoprotein cholesterol; Non-HDL-C, non high density lipoprotein cholesterol; UCB, umbilical cord blood.

needed to understand the role of dyslipidemia in the pathogenesis of this disease. ${ }^{9}$

The associations found between maternal blood lipid parameters (TG and non-HDL-C) and elevated UtA-PI suggest that a markedly high level of TG may potentially cause deterioration of the uteroplacental blood flow, especially in the case of preeclampsia. The high serum TG level, as an important cardiovascular risk marker, has been proposed to be independently associated with endothelial dysfunction in preeclamptic women. ${ }^{10,21}$ Along with hypertriglyceridemia, oxidative stress is also considered another process associated with endothelial dysfunction in preeclampsia. ${ }^{10,22}$ Furthermore, a prospective study including 19 women with mild and 15 women with severe preeclampsia showed that severe preeclampsia is associated with oxidative stress, which may impair the placental perfusion measured by the increase of UtA resistance. ${ }^{22}$

According to recent findings, LDL-C is not the only form of cholesterol linked with adverse events. Studies have shown that atherogenic lipoproteins, referred to as non-HDL have a better predictive value for cardiovascular events than LDL-C levels only. ${ }^{23,24}$ Interestingly, a significant correlation was found between disturbed uterine perfusion and increased nonHDL-C levels in the current study. This finding could show the "atherogenic" role of non-HDL-C. This pro-atherogenic state in preeclampsia could lead to lipid deposition in the walls of the UtA extending to the spiral arteries. These vascular lesions resemble early stages of atherosclerosis and are thought to play a critical role in pathological vascular remodeling, ${ }^{25}$ leading to an increased UtA-PI and the subsequent adverse outcomes due to uteroplacental insufficiency. ${ }^{26}$

In a study by Bugatto et al, ${ }^{12}$ it was observed that maternal serum TG, HDL, and TC/HDL ratio were significantly associated with disturbed uterine blood flow in pregnancies complicated with GDM, but no association was seen in the control group. However, no study has so far investigated the association between lipid markers and uteroplacental flow in HDP.

Preceding studies have emphasized that vascular and hemodynamic function could be partially programmed 
during fetal life and that this background could be significantly involved in the arterial stiffening, and process of vascular remodeling in later life. ${ }^{27,28}$ Consequently, evaluating fetal vessels indirectly through Doppler strategies might be an advantageous technique to detect abnormal blood flow since vascular wall cells undergo metabolic and hormonal changes during the fetal life period. ${ }^{29}$

Despite several studies evaluating lipid parameters in maternal serum and its association with adverse outcomes, little is known about fetal lipid parameters and their role in different types of pathologies. ${ }^{30,31}$ Rodie et $\mathrm{al}^{30}$ reported a significant increase in levels of TG, TC, and TC/HDL-C ratio in UCB of fetuses of preeclamptic women, but Ophir et $\mathrm{al}^{31}$ did not find such an association and they only observed a slight increase of LDL-C in UCB of preeclamptic pregnancies. Among lipid parameters measured in newborns of our study population, elevated UCB TG concentration was shown to be associated with abnormal UA-PI and MCA-PI in the HDP group. This association pattern showed a similar trend when newborns of preeclamptic women were analyzed separately. Currently, a theoretical explanation provided by Romanowicz et $\mathrm{al}^{32}$ suggested that cholesterol accumulation in the umbilical artery wall may be responsible for the "early aging" of the umbilical cord tissue and consequently trigger the development of hypertension; however, the potential mechanisms still deserve further exploration.

Overall, our study results suggest that HDP is a proatherogenic state, which is mainly attributable to preeclampsia, and elevated lipid levels may play a significant role in disturbing maternal-fetal circulation. In addition, further research should be conducted to study the mechanisms of action of lipids in maternal-fetal blood circulation in depth.

Although this study is the first of its kind to analyze the association between lipid profiles of mother-newborn pairs and uterine and fetal placental blood flow, there are also limitations that should be highlighted: 1) we were not able to adjust for serum markers of inflammation and oxidative stress and these two factors could have confounded our results by affecting uterine blood flow especially in the case of preeclampsia; 2) although all maternal blood samples were collected in the fasting state, a few UCB samples were collected in non-fasting conditions due to emergency cesarean section; and 3) only a small number of patients developed preeclampsia $(n=80)$ or gestational hypertension $(\mathrm{n}=90)$.

\section{Conclusion}

In summary, our study supports the association of maternal TG and non-HDL-C with abnormal uterine blood flow waveforms in women with HDP. Additionally, we found that fetal hypertriglyceridemia was the only fetal lipid abnormality associated with disturbed blood flow in UA and MCA. We suggest that affected mothers and their children could be targeted for programs aimed at reducing severe clinical cardiovascular diseases, by lifestyle or pharmacological interventions.

\section{Abbreviations}

HDP, hypertensive disorders of pregnancy; UCB, umbilical cord blood; TG, triglyceride; TC, total cholesterol; HDL-C, high-density lipoprotein cholesterol; LDL-C, low-density lipoprotein cholesterol; Non-HDL-C, non- high-density lipoprotein cholesterol; UtA, uterine artery; UA: umbilical artery; MCA: middle cerebral artery; PI: pulsatility index; GDM: gestational diabetes mellitus; IUGR: intrauterine fetal growth restriction; AOR: adjusted odds ratio.

\section{Data Sharing Statement}

The datasets analyzed during the current study are available from the corresponding author on reasonable request.

\section{Acknowledgment}

This study was extracted from the thesis written by Kamran Hessami for an MD degree (code No. 97-01-0118902).

\section{Author Contributions}

All authors contributed to data analysis, drafting or revising the article, gave final approval of the version to be published, and agree to be accountable for all aspects of the work.

\section{Funding}

The project was financed by Vice Chancellor for Research of the Shiraz University of Medical Science, Shiraz, Iran (Grant No. 97-01-01-18902). The funding organization played no role in the study design; in the collection, analysis, and interpretation of data; in the writing of the report; or in the decision to submit the report for publication.

\section{Disclosure}

The authors report no conflicts of interest in this work. 


\section{References}

1. Wang C, Kong L, Yang Y, et al. Recommended reference values for serum lipids during early and middle pregnancy: a retrospective study from China. Lipids Health Dis. 2018;17(1):246. doi:10.1186/s12944018-0885-3

2. Brizzi P, Tonolo G, Esposito F, et al. Lipoprotein metabolism during normal pregnancy. Am J Obstet Gynecol. 1999;181(2):430-434. doi:10.1016/S0002-9378(99)70574-0

3. Nasioudis D, Doulaveris G, Kanninen TT. Dyslipidemia in pregnancy and maternal-fetal outcome. Minerva Ginecol. 2019;71(2):155-162. doi:10.23736/S0026-4784.18.04330-7

4. Jin WY, Lin SL, Hou RL, et al. Associations between maternal lipid profile and pregnancy complications and perinatal outcomes: a population-based study from China. BMC Pregnancy Childbirth. 2016;16:60. doi:10.1186/s12884-016-0852-9

5. Serrano NC, Guio-Mahecha E, Quintero-Lesmes DC, et al. Lipid profile, plasma apolipoproteins, and pre-eclampsia risk in the GenPE case-control study. Atherosclerosis. 2018;276:189-194. doi:10.1016/j.atherosclerosis.2018.05.051

6. Ziaei S, Bonab KM, Kazemnejad A. Serum lipid levels at 28-32 weeks gestation and hypertensive disorders. Hypertens Pregnancy. 2006;25(1):3-10. doi:10.1080/10641950500543756

7. Jan MR, Nazli R, Shah J, Akhtar T. A study of lipoproteins in normal and pregnancy induced hypertensive women in tertiary care hospitals of the north west frontier province-Pakistan. Hypertens Pregnancy. 2012;31(2):292-299. doi:10.3109/10641955.2010.507843

8. Ghio A, Bertolotto A, Resi V, Volpe L, Di Cianni G. Triglyceride metabolism in pregnancy. Adv Clin Chem. 2011;55:133-153.

9. Spracklen CN, Smith CJ, Saftlas AF, Robinson JG, Ryckman KK. Maternal hyperlipidemia and the risk of preeclampsia: a meta-analysis. Am J Epidemiol. 2014;180(4):346-358. doi:10.1093/ aje/kwu 145

10. Kaaja R. Lipid abnormalities in pre-eclampsia: implications for vascular health. Clin Lipidol. 2011;6(1):71-78. doi:10.2217/clp.10.82

11. Charlton F, Tooher J, Rye KA, Hennessy A. Cardiovascular risk, lipids and pregnancy: preeclampsia and the risk of later life cardiovascular disease. Heart Lung Circ. 2014;23(3):203-212. doi:10.1016/ j.hlc.2013.10.087

12. Bugatto F, Quintero-Prado R, Visiedo FM, et al. The influence of lipid and proinflammatory status on maternal uterine blood flow in women with late onset gestational diabetes. Reprod Sci. 2018;25 (6):837-843. doi:10.1177/1933719117698576

13. Joern H, Funk A, Rath W. Doppler sonographic findings for hypertension in pregnancy and HELLP syndrome. J Perinat Med. 1999;27 (5):388-394. doi:10.1515/JPM.1999.053

14. Abdelwahid HH, Wahab BA, Mahmoud MZ, Abukonna A, Saeed Taha EA. Effects of gestational hypertension in the pulsatility index of the middle cerebral and umbilical artery, cerebro-placental ratio, and associated adverse perinatal outcomes. J Radiat Res Appl Sci. 2018;11(3):195-203. doi:10.1016/j.jrras.2018.02.001

15. Lopez-Mendez MA, Martinez-Gaytan V, Cortes-Flores R, et al. Doppler ultrasound evaluation in preeclampsia. BMC Res Notes. 2013;6(1):477. doi:10.1186/1756-0500-6-477

16. Pirhonen J, Bergersen TK, Abdlenoor M, Dubiel M, Gudmundsson S. Effect of maternal age on uterine flow impedance. J Clin Ultrasound. 2005;33(1):14-17. doi:10.1002/(ISSN)1097-0096

17. Grundy SM, Stone NJ, Bailey AL, et al. 2018 AHA/ACC/AACVPR/ AAPA/ABC/ACPM/ADA/AGS/APhA/ASPC/NLA/PCNA guideline on the management of blood cholesterol: a report of the American College of Cardiology/American Heart Association task force on clinical practice guidelines. Circulation. 2019;139(25):e1082-e1143. doi:10.1161/CIR.0000000000000625
18. Baschat AA, Gembruch U. The cerebroplacental Doppler ratio revisited. Ultrasound Obstet Gynecol. 2003;21(2):124-127. doi:10.1 002/uog. 20

19. Gómez O, Figueras F, Fernández S, et al. Reference ranges for uterine artery mean pulsatility index at 11-41 weeks of gestation. Ultrasound Obst Gynecol. 2008;32(2):128-132. doi:10.1002/uog. v32:2

20. Ciobanu A, Wright A, Syngelaki A, Wright D, Akolekar R, Nicolaides KH. Fetal medicine foundation reference ranges for umbilical artery and middle cerebral artery pulsatility index and cerebroplacental ratio. Ultrasound Obstet Gynecol. 2019;53(4):465-472. doi:10.1002/uog.2019.53.issue-4

21. Sattar N, Bendomir A, Berry C, Shepherd J, Greer IA, Packard CJ. Lipoprotein subfraction concentrations in preeclampsia: pathogenic parallels to atherosclerosis. Obstet Gynecol. 1997;89(3):403-408. doi:10.1016/S0029-7844(96)00514-5

22. Kornacki J, Kozlik J, Dubiel M, Skrzypczak J. Estimation of oxidative stress and its correlation with uterine arteries Doppler velocimetry in women with preeclampsia. Ginekol Pol. 2004;75(9):681-691.

23. Wen J, Huang Y, Lu Y, Yuan H. Associations of non-high-density lipoprotein cholesterol, triglycerides and the total cholesterol/ HDL-c ratio with arterial stiffness independent of low-density lipoprotein cholesterol in a Chinese population. Hypertens Res. 2019;42 (8):1223-1230. doi:10.1038/s41440-019-0251-5

24. Bergmann K. Non-HDL cholesterol and evaluation of cardiovascular disease risk. EJIFCC. 2010;21(3):64-67.

25. Staff AC, Dechend R, Pijnenborg R. Learning from the placenta: acute atherosis and vascular remodeling in preeclampsia-novel aspects for atherosclerosis and future cardiovascular health. Hypertension. 2010;56(6):1026-1034. doi:10.1161/HYPERTENSIO NAHA. 110.157743

26. Olofsson P, Laurini RN, Marsal K. A high uterine artery pulsatility index reflects a defective development of placental bed spiral arteries in pregnancies complicated by hypertension and fetal growth retardation. Eur J Obstet Gynecol Reprod Biol. 1993;49 (3):161-168. doi:10.1016/0028-2243(93)90265-E

27. Nilsson PM, Lurbe E, Laurent S. The early life origins of vascular ageing and cardiovascular risk: the EVA syndrome. J Hypertens. 2008;26(6):1049-1057. doi:10.1097/HJH.0b013e3282f82c3e

28. Singhal A, Lucas A. Early origins of cardiovascular disease: is there a unifying hypothesis? Lancet. 2004;363(9421):1642-1645. doi:10.1016/S0140-6736(04)16210-7

29. Blanco MV, Vega HR, Giuliano R, et al. Histomorphometry of umbilical cord blood vessels in preeclampsia. J Clin Hypertens. 2011;13(1):30-34. doi:10.1111/jch.2011.13.issue-1

30. Rodie VA, Caslake MJ, Stewart F, et al. Fetal cord plasma lipoprotein status in uncomplicated human pregnancies and in pregnancies complicated by pre-eclampsia and intrauterine growth restriction. Atherosclerosis. 2004;176(1):181-187. doi:10.1016/j.atherosclerosis. 2004.04.026

31. Ophir E, Dourleshter G, Hirsh Y, Fait V, German L, Bornstein J. Newborns of pre-eclamptic women: a biochemical difference present in utero. Acta Obstet Gynecol Scand. 2006;85(10):1172-1178. doi:10.1080/00016340600697272

32. Romanowicz L, Bańkowski E. Preeclampsia-associated alterations in sphingolipid composition of the umbilical cord artery. Clin Biochem. 2009;42(16-17):1719-1724. doi:10.1016/j.clinbiochem.2009.07.011 


\section{Publish your work in this journal}

The International Journal of Women's Health is an international, peerreviewed open-access journal publishing original research, reports, editorials, reviews and commentaries on all aspects of women's healthcare including gynecology, obstetrics, and breast cancer. The

manuscript management system is completely online and includes a very quick and fair peer-review system, which is all easy to use. Visit http://www.dovepress.com/testimonials.php to read real quotes from published authors.

Submit your manuscript here: https://www.dovepress.com/international-journal-of-womens-health-journal 